\title{
Single-incision vaginal approach to treat cystocele and vault prolapse with an anterior wall mesh anchored apically to the sacrospinous ligaments
}

\author{
Robert D. Moore • Gretchen K. Mitchell • \\ John R. Miklos
}

Received: 21 February 2011 / Accepted: 11 July 2011 / Published online: 25 August 2011

(C) The Author(s) 2011. This article is published with open access at Springerlink.com

\begin{abstract}
Introduction and hypothesis The safety and early efficacy of a new technique to treat cystocele and/or concomitant apical prolapse through a single vaginal incision with a lightweight mesh anchored apically bilaterally to the sacrospinous ligaments is reported.

Methods Women with anterior compartment and/or apical prolapse $\geq$ stage II underwent repair through a single anterior vaginal wall incision with the Anterior Elevate System (AES). The technique utilizes a lightweight ( $24 \mathrm{~g} /$ $\mathrm{m}^{2}$ ) type I mesh anchored to the sacrospinous ligaments via two mesh arms with small self-fixating tips. The bladder neck portion of the graft is anchored to the obturator internus with similar self-fixating tips. The apical portion of the graft is adjustable to vaginal length prior to locking in place. Outcome measures included prolapse degree at last follow-up visit, intra/post-operative complications, and QOL assessments.

Results Sixty patients were implanted with average followup of 13.4 months (range 3-24 months). Mean pre-op Ba was $+2.04 \pm 1.3$ and $\mathrm{C}-2.7 \pm 2.9$. Average blood loss was $47 \mathrm{cc}$ and average hospital stay was $23 \mathrm{~h}$. Sixty-two percent of patients had concomitant sling for SUI. Mean post-op Ba is $-2.45 \pm 0.9$ and $C-8.3 \pm 0.9$. There was no statistical difference in pre- to post-op TVL. Objective cure rate at current follow-up is $91.7 \%$ (sstage 1). To date, there have been no mesh extrusions. No patients have reported significant buttock or leg pain. No patients have required surgical revision for any reason.
\end{abstract}

R. D. Moore $(\bowtie) \cdot$ G. K. Mitchell $\cdot$ J. R. Miklos

Atlanta Urogynecology Associates,

Atlanta, GA, USA

e-mail: rdm@miklosandmoore.com

URL: www.atlurogyn.com
Conclusion The AES is a minimally invasive technique to treat anterior compartment and/or apical prolapse through a single vaginal incision. Initial results show the procedure to be safe and early efficacy is promising. Longer-term followup is ongoing.

Keywords Cystocele $\cdot$ Vaginal mesh $\cdot$ Mesh complications Elevate $\cdot$ Sacrospinous ligament

\section{Introduction}

Pelvic organ prolapse (POP) is a significant health issue in females worldwide $[1,2]$. There are approximately 250,000 procedures annually in the USA for POP, with as many as $29 \%$ of women having to undergo repeat surgery [2]. Traditional anterior repair of cystocele or anterior compartment prolapse utilizing the patient's own tissue is a compensatory procedure that utilizes weakened and/or damaged tissue and has reported failure rates in the range of 40-60\% [3]. Additionally, plication or colporrhaphy techniques address only midline defects in the anterior compartment and do not provide any apical support which may also contribute to the failure rates seen with this type of repair [3].

Abdominal Y-mesh sacralcolpopexy has the highest cure rates in the literature for vault prolapse, and the benefit of utilizing mesh in this particular repair seems to outweigh the risks [4]. It results in anatomic repair with minimal tension and does not rely on the patients weakened tissue to maintain support. With the success of apical graft use, more recently, graft augmentation of prolapse repair has been utilized via the vaginal route. A recent Cochrane review has confirmed that mesh use in the anterior compartment has a lower failure rate versus traditional repair $[5,6]$. Mesh trocar-based kits such as Perigee and Prolift were developed 
to try to simplify the placement of mesh, and initial studies have shown cure rates in the range of 87-96\% [7-9]. These first-generation kits all required needles to be passed through the groins and obturator space with mesh arms subsequently pulled through this same space to attach the body of the graft to the levator muscles. Although many studies have shown successful cure rates when used by experienced surgeons in the right patient population, complications resulting from the external needle passes such as visceral and vascular injury as well as postoperative mesh complications have been concerning [911]. Vaginal or pelvic pain from the mesh arms being too tight, as well as issues such as rates of mesh extrusion as high as $15 \%$, have been reported [12-14]. Additionally, a shortfall of most of these first-generation kits, is that they did not offer true level I or apical support in the anterior compartment, which may have led to apical failure.

In an attempt to both benefit from the advantages of mesh use in the anterior compartment and also decrease some of the associated risks seen in the first-generation kits, a new singleincision vaginal approach was developed that utilizes a lighter and less-dense type I polpypropylene mesh (Intepro Lite, AMS) and eliminates all blind external needle passes. It also obtains true level I support through an anterior approach, by attaching the mesh, in an adjustable, tension-free matter to the sacrospinous ligaments via a novel and very minimally invasive fixation technique involving small self-fixating tips attached to the apical mesh arms. The purpose of the current study is to report the outcomes on a group of patients that underwent this procedure for treatment of symptomatic anerior compartment and/or apical prolapse at our specialty urogynecology center.

\section{Materials and methods}

This study is a descriptive retrospective case series of 60 consecutive women with symptomatic stage 2 or greater anterior compartment prolapse (cystocele) that underwent anterior repair with mesh graft augmentation and vaginal vault suspension through a single transvaginal incision utilizing the Anterior Elevate by AMS (American Medical Systems, Minnetonka, MN, USA) system over a consecutive 12-month period at our center. Comprehensive preoperative urogynecologic exams were completed including prolapse quantification utilizing the International Continence Society Pelvic Organ Prolapse Quantification (POPQ) staging system. Inclusion criteria were patients with symptomatic anterior compartment prolapse $\geq$ stage 2 that received the Anterior Elevate procedure over a consecutive 12-month timeframe at our center. Exclusion criteria included any patient that had any other vault suspension procedure at the time of the surgery. Additional testing included complex urodynamic testing to evaluate for the presence of concomitant stress urinary incontinence (SUI) (or detrusor instability) with and without the patient's prolapse reduced. If SUI was documented on urodynamic testing with or without the prolapse reduced, the patient was scheduled for a sub-urethral mesh tape sling procedure at the time of surgery. A sling was not placed prophylactically in any patient if SUI was not seen on testing.

\section{Procedure technique}

The procedure is begun with injection of a hydrodissection solution (25 cc of 1\% lidocaine with epinephrine 1:200,000 diluted in $250 \mathrm{cc}$ of saline) into the anterior vaginal wall. Typically, 40-60 cc of this solution is utilized. A 3 to $4-\mathrm{cm}$ vertical incision is then made in the anterior vaginal wall starting at the level of the bladder neck towards the vaginal vault or the cervix. The incision is kept as small as possible and is NOT taken all the way to the apex of vagina. If the uterus was in situ, it was left in place in the current trial. A full-thickness dissection is then completed in the relatively avascular vesico-vaginal space out to the pelvic sidewalls and then up to the ischial spines bilaterally. Once the spine is reached, a medial sweep with the index and/or middle finger is completed to isolate the sacropinous ligament (SSL). It should be noted that the retropubic space does not need to be entered, nor does the ligament need to be visualized. The bladder should also be dissected off of the cervix or vaginal vault. The bladder neck arms of the graft are placed into the obturator internus muscle at the level of the bladder neck with their small self-fixating tips. The separate apical arms are then placed in the SSLs bilaterally approximately $2 \mathrm{~cm}$ medial to the ischial spine (Fig. 1). The apical portion of the graft is then fed over the arms attached

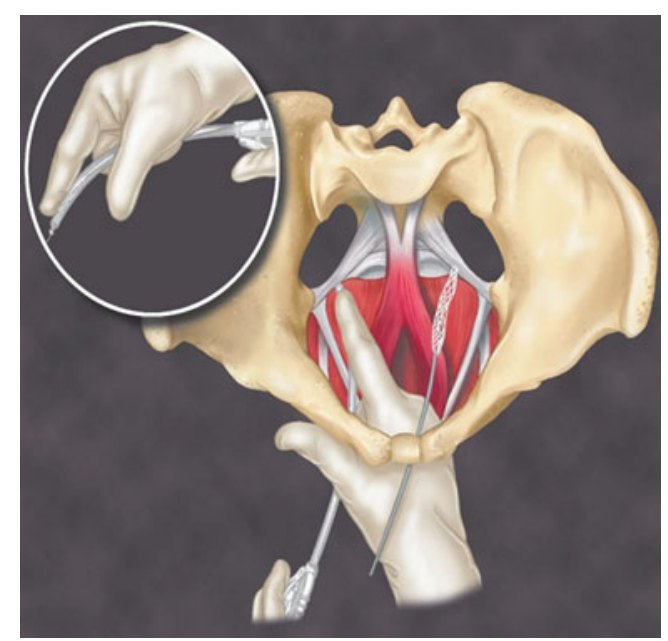

Fig. 1 Apical arm of the Elevate system placed into the sacropinous ligament $2 \mathrm{~cm}$ medial to the ischial spine. (Reproduced with permission from AMS) 
to the SSLs and the vault adjusted into place in a tension free manner with an adjusting tool provided. Two 2-0 PDS sutures are used to attach the graft in the midline to the pericervical ring or the vaginal cuff. Once the final adjustment is reached, the graft is locked in place with small locking eyelets and the excess mesh cut off of the apical arms. Minimal to no vaginal epithelium is excised and the incision closed with a 2-0 delayed absorbable suture in a running-locked fashion. Cystoscopy is completed, foley catheter and vaginal packing placed for $24 \mathrm{~h}$.

If an incontinence procedure was completed concomitantly, a separate sub-urethral incision was made and the tension-free sling placed utilizing standard technique. Remaining prolapse procedures were then completed as necessary. If stable, patients were discharged home on postoperative day 1. Vaginal estrogen cream was started 1 week post-operatively and used every other day.

Patients were evaluated in the office at 4 weeks, 3 months, 6 months and then every 6 months thereafter. ICS POP-Q staging was completed as well as subjective assessment of prolapse (feeling or seeing a bulge), incontinence and urinary urgency and frequency symptoms. Quality of life questionnaires utilized were the Incontinence Impact Questionnaire-Short form (IIQ-7) and the Urogenital Distress Inventory-Short form (UDI-6). Objective cure was defined if the midline anterior vaginal wall (points Aa and $\mathrm{Ba}$ ) was $<1.0-1.0 \mathrm{~cm}$ inside the hymenal ring and the apex was also less than or equal to stage I.

\section{Results}

Patient demographics are presented in Table 1. Of the 60 patients, $11(18.4 \%)$ had stage 2 prolapse, 43(71.6\%) stage 3 , and $6(10 \%)$ had stage 4 on pre-operative pelvic examination and POP-Q scoring. The mean $\mathrm{Ba}$ value $( \pm \mathrm{SD})$ was $+2.04 \pm 1.3 \mathrm{~cm}$ outside the vaginal opening, average $C$ value $( \pm \mathrm{SD})$ was $-2.7 \pm 2.9$. Sixteen $(26.6 \%)$ patients had previous anterior vaginal wall repairs and had recurrent cystoceles. Seventy-seven percent of patients had clinically significant apical prolapse. SUI was present in $61.6 \%$ of patients and tension-free tape slings were placed at the time of their surgeries. Twenty-six patients (43.3\%) presented with SUI and $11(18.3 \%)$ were discovered to have occult stress leakage with their prolapse reduced during urodynamic testing. Concomitant procedures at time of surgery included, 26/60 patients $(43.3 \%)$ with posterior repair (16 with mesh grafts, 10 no graft used). No patient had any other vault support besides the Anterior Elevate and no patient underwent hysterectomy $(27 \%$ of patients had their uterus in situ) (Table 2$)$. Nineteen patients $(31.6 \%)$ were sexually active prior to surgery.
Table 1 Demographics

\begin{tabular}{lc}
\hline$N=60$ & \\
\hline Age (years \pm SD) & $70.8 \pm 6.1$ (range $51-81)$ \\
Parity & $2.8 \pm 1.6$ (range 0-8) \\
Previous hysterectomy & $N=44(73 \%)$ \\
Menopausal & $N=59(98.4 \%)$ \\
Estrogen use & $N=19(31.6 \%)$ \\
Previous repair & $N=16(26.6 \%)$ \\
$>1$ Previous repair & $N=5(8.3 \%)$ \\
Prolapse stage & \\
II & $N=11(18.4 \%)$ \\
III & $N=43(71.6 \%)$ \\
IV & $N=6(10.0 \%)$
\end{tabular}

Average blood loss was $47 \mathrm{cc}$ (range $25-125 \mathrm{cc}$ ) and was deemed minimal. There were no post-operative bleeds or hematomas and no patient had to be taken back to the O.R. for bleeding or pain. There was one midline cystotomy above the trigone that occurred during the dissection of the anterior wall. This was closed in a two-layer fashion with absorbable sutures and the mesh still placed and ureteral patency confirmed. The average hospital stay was $23 \mathrm{~h}$ (range $18 \mathrm{~h}$ to 2 days). Average time to void was 3.6 days (range 1-10 days). Foley catheters were taken out on postoperative day 1 and voiding trial attempted. If patient did not pass the voiding trial, she was sent home with an indwelling catheter and voiding trial re-attempted on postoperative day 3 . Twelve patients required catheterization for more than 3 days with ten of these having sling procedure at time of surgery. One patient who had the complication of cystotomy had her catheter for 10 days and had no sequalae from the injury. Four patients suffered from post-operative urinary tract infections (UTI); however, one of these had a history of recurrent UTIs.

Average follow-up was 13.4 months (range 324 months). Only one patient has symptoms of recurrent prolapse for a subjective cure rate of $98.4 \%$. Objective/ anatomic cure rate was $91.7 \%$ using a definition of $\mathrm{Ba}<$
Table 2 Concomitant procedures

\begin{tabular}{lrc}
\hline Procedure & $n$ & $\%$ \\
\hline Hysterectomy & 0 & 0 \\
Sling & & \\
Mini-sling & 33 & 55 \\
TOT & 2 & 3.3 \\
TVT & 2 & 3.3 \\
Posterior repair & & \\
No graft & 10 & 16.6 \\
Mesh graft & 16 & 26.6 \\
\hline
\end{tabular}


-1.0 ( $\leq$ stage I) and no patients have required repeat surgery for prolapse. Mean Ba value was $-2.45 \pm 0.9$, point $C$ was $-8.3 \pm 0.9$, and TVL was $9.1 \pm 0.3 \mathrm{~cm}$. Mean vaginal length did not change statistically from pre-operative values (Table 3).

There have been no patients that reported any significant buttock or leg pain that would be consistent with a pudendal or obturator type of neuropathy. Three patients suffered from post-operative levator myalgia or vaginal pain that required short-term treatment with NSAIDS and/ or physical therapy with resolution of all patients' pain. In this predominantly elderly post-menopausal patient group, $31 \%$ were sexually active pre-operatively with one of these patients reporting clinically significant dyspareunia that is being treated with conservative measures (physical therapy) and is improving.

Thirty-two patients (53\%) complained of significant urge symptoms pre-operatively, and 20 of these patients (62\%) had resolution of these symptoms post-operatively following their surgery. One patient $(1.6 \%)$ developed de-novo urge symptoms post-operatively requiring treatment with anticholinergic agents. Overall IIQ-7 and UDI-6 scores improved significantly from baseline to follow-up (Table 4). The UDI-6 subdomains of urge, stress, and obstructive symptoms were also evaluated and a significant improvement in each domain was also seen in the overall group (Table 4). Patients that had a concomitant sling procedure or that had a previous repair did not statistically differ in improvement in these QOL indices compared to those that did not (Tables 5 and 6). The UDI-6 subdomain scores were also evaluated in the patients that had a concomitant sling or not and these results can be seen in Table 6 .

Three patients suffered from significant SUI postoperatively. One had a concomitant tension-free sling (TVT type) at time of surgery that failed and the other two did not have a concomitant sling as they did not suffer from SUI pre-operatively and tested negative on UDTs. The patient that had the sling already placed is scheduled for treatment with injectable bulking agents and the other two are being scheduled for outpatient sling procedures.

There have been no mesh extrusions noted to date. There were no post-operative infections of the mesh and no mesh had to be removed secondary to infection or pain or allergic reaction. No patients had to be taken back to the operating room for revision of the mesh or release of any of the mesh attachment points for pain or dyspareunia.

\section{Discussion}

The current series utilizing the Anterior Elevate system, showed excellent anatomic results and an objective cure rate of $92 \%$ with up to 24 months of $\mathrm{f} / \mathrm{u}$ (mean $\mathrm{f} / \mathrm{u}$ 13.4 months). Subjectively, only one patient complained of symptomatic prolapse ( $98.4 \%$ subjective cure rate) and no patient has had prolapse of the anterior wall outside of the introitus. These results are consistent with other series utilizing synthetic mesh for anterior compartment repair; however, we believe the Anterior Elevate procedure to be less invasive and a more simplified technique to place an anterior wall graft. Additionally, it allows for concomitant apical support at the time of anterior compartment repair. Goldberg et al. showed the anterior approach to SSL to result in anatomic restoration of the vaginal vault with cure rates consistent with the posterior approach [15] which our anatomic results support this as well.

The Anterior Elevate system utilizes small self-fixating tips for attachment of the graft to the obturator internus muscle at the level of the bladder neck and into the SSLs at the apex (Figs. 2 and 3). The attachment of the graft to the SSLs involves minimal dissection with placement of the small tip into the mid-portion of the ligament. Secondary to this, risk of nerve injury or post-operative pain pudendal nerve type pain syndromes is minimal and this is supported in the current trial with no patients developing a postoperative pudendal nerve pain type syndrome and no patient requiring any surgical intervention for postoperative pain or dyspareunia. The elimination of lateral mesh arms penetrating through and through the levators also should help reduce the risk of vaginal or pelvic pain from the arms being placed too tight and "banding" in the vagina. This and/or mesh "bunching" seems to be the cause of most cases of dyspareunia with the first generation trocar-based mesh kits $[12,13,16]$. We did have three
Table 3 Pre-operative versus post-operative POP-Q measurements (mean)

\begin{tabular}{lccc}
\hline & Preoperative & Postoperative & $P$ value $(t$ test $)$ \\
\hline Mean POP-Q measurements & & & \\
Point Aa (cm) & $+1.4 \pm 1.4$ & $-2.4 \pm 0.8$ & $<0.001$ \\
Point Ba (cm) & $+2.0 \pm 1.3$ & $-2.5 \pm 0.9$ & $<0.001$ \\
Point C (cm), cervix & $-2.7 \pm 2.9$ & $-8.3 \pm 0.9$ & $<0.001$ \\
Point Ap & $-1.1 \pm 1.3$ & $-2.4 \pm 0.6$ & $<0.001$ \\
Point Bp & $-0.9 \pm 1.6$ & $-2.3 \pm 0.6$ & $<0.001$ \\
Total vaginal length $(\mathrm{cm})$ & $9.09 \pm 0.5$ & $9.16 \pm 0.3$ & 0.343 \\
\hline
\end{tabular}


Table 4 Overall UDI-6 and IIQ-7 scores (Pre-operative vs. Post-operative) and UDI-6 subscales

\begin{tabular}{|c|c|c|c|c|}
\hline Variable $(n=58)^{\mathrm{a}}$ & Baseline mean $\pm \mathrm{SD}$ & Follow-up mean \pm SD & Follow-up vs. baseline & $P$ value ( $t$ test) \\
\hline UDI-6 & $42.9 \pm 26.9$ & $22.4 \pm 24.4$ & $-20.6 \pm 30.8$ & $<0.001$ \\
\hline IIQ-7 & $31.5 \pm 25.7$ & $15.4 \pm 23.4$ & $-16.1 \pm 26.9$ & $<0.001$ \\
\hline \multicolumn{5}{|l|}{ UDI subscales } \\
\hline Irritative (56) & $52.4 \pm 31.7$ & $26.9 \pm 32.3$ & $-24.4 \pm 38.5$ & $<0.001$ \\
\hline Stress (56) & $44.6 \pm 29.8$ & $25.4 \pm 30.4$ & $-20.2 \pm 36.5$ & $<0.001$ \\
\hline Obstructive (58) & $40.4 \pm 30.4$ & $15.5 \pm 22.7$ & $-24.1 \pm 32.9$ & $<0.001$ \\
\hline
\end{tabular}

a $58 / 60$ patients filled out both pre- and post-operative questionnaires

patients develop post-operative pain remote from surgery that required treatment; however, it was all deemed muscular and responded to pelvic floor physical therapy and did not need surgical intervention. This percentage is consistent with other forms of prolapse treatment. One does need to be careful; however, in the adjustment of the graft at the apex as if too much tension is placed then this could potentially cause pain that may require release.

Concerning the safety of the procedure, bleeding was minimal in most cases with average blood loss at $47 \mathrm{cc}$. No patients developed post-operative hematoma nor did any require blood transfusion. One patient suffered a midline cystotomy during the procedure; however, this was during the dissection of the anterior wall and was not related to the mesh or needle passes. We repaired the cystotomy with a double-layered closure, still placed the mesh, and she recovered without sequelae. Overall, with the elimination of blind external needle passes the risk of intra-operative bladder or vascular injury is reduced.

Our mesh extrusion rate in the current trial was very low, and we attribute that to two variables: mesh quality and surgical technique. The mesh used in the current trial (Intepro Lite) is a type I macroporous polypropylene mesh that is $50 \%$ less dense and lighter than the first generation type I mesh (Intepro) used in Apogee/Perigee. In a previously published trial from our center, we reported mesh extrusion rate of $6.5 \%$ with the use of the Perigee procedure for treatment of cystocele in a similar group of patients [17], which is consistent with other reports in the literature. Utilizing a less dense and softer mesh, results in a smaller mesh load and, most likely, less inflammatory response during healing which may reduce mesh extrusion rates. Additionally, our surgical technique of utilizing a deeper dissection plane, i.e., a full-thickness dissection utilizing hydrodissection in a more avascular space, allows for less bleeding during the procedure, less risk of postoperative hematoma, and a thicker vascular flap healing over the mesh. All of these factors may contribute to the lower extrusion rate seen in our trial. Other lighter meshes have also been introduced, however, have not shown this low of extrusion rate. Prolift-M utilizes a soft polypropylene mesh that has a similar density to Intepro-Lite however is $50 \%$ Monocryl, and therefore, $50 \%$ of the mesh is absorbable. The thought was that this would decrease vaginal exposure rate; however, in a recent study, Cosson et al. reported a relatively high extrusion rate of $10.5 \%$ [18].

Sixteen patients $(26.6 \%)$ had previous repairs and would be considered higher risk for failure; however, their cure rate was consistent with patients that had not had prior repair and there was no difference in complications seen in these patients either. In our opinion, this is a group of patients that benefit the greatest from a graft, given a previous failure using their own tissue, and the results of these patients in our series is very encouraging.

The current study is limited by its retrospective nature and its medium-term follow-up. Another limitation, again inherent to a retrospective single-center trial, is surgeon bias in evaluating their own surgical outcomes. A limitation of any surgical trial that also has to be considered is surgeon experience with the particular procedure or similar procedures as well as the anatomy of the dissection involved.

Table 5 IIQ-7/UDI-6 scores in patients with previous anterior compartment repairs compared to those that did not

\begin{tabular}{llcccccc}
\hline \multicolumn{2}{l}{ Previous repair } & IIQ-7 Pre & IIQ-7 Post & IIQ-7 Change & UDI-6 Pre & UDI Post & UDI-6 Change \\
\hline \multirow{2}{*}{ No=54 } & Mean & 29.3 & 10.6 & -18.7 & 27.7 & 13.6 & -14.1 \\
& StDev & 26.0 & 16.8 & 25.0 & 19.0 & 15.3 & 23.4 \\
\multirow{2}{*}{ Yes=16 } & Mean & 36.4 & 28.1 & -8.3 & 43.5 & 25.6 & -17.9 \\
& StDev & 25.7 & 32.9 & 31.3 & 19.7 & 23.4 & 23.2 \\
& $T$ test (between groups) & 0.35 & 0.01 & 0.19 & 0.01 & 0.02 & 0.58 \\
\hline
\end{tabular}


Table 6 IIQ-7/UDI-6 scores (and UDI-6 domain scores) in patients with and without concomitant sling at time of surgery

\begin{tabular}{lcccr}
\hline Questionnaire $(n)$ & Pre-operative score & Post-operative score & Mean improvement & $P$ value \\
\hline IIQ-7 & & & & \\
Sling (37) & $33.2 \pm 26.0$ & $16.6 \pm 24.6$ & $16.6 \pm 28.8$ & 0.001 \\
No sling (21) & $28.1 \pm 26.0$ & $13.6 \pm 22.1$ & $14.5 \pm 24.3$ & 0.013 \\
$T$ test(between groups) & 0.48 & 0.65 & 0.72 & \\
UDI-6 & & & & \\
Sling (38) & $45.1 \pm 23.6$ & $26.0 \pm 27.0$ & $19.0 \pm 28.6$ & $<0.001$ \\
No sling (22) & $38.5 \pm 32.2$ & $16.4 \pm 19.0$ & 0.72 & \\
$T$ test(between groups) & 0.37 & 0.15 & & \\
UDI subscales & & & $26.6 \pm 35.1$ & \\
Irritative & & & $20.6 \pm 40.8$ & 0.031 \\
Sling (37) & $53.2 \pm 31.4$ & $26.8 \pm 33.5$ & & \\
No sling (21) & $48.4 \pm 32.9$ & $27.3 \pm 31.1$ & $19.9 \pm 38.4$ & 0.004 \\
Stress & & & $20.8 \pm 33.7$ & 0.012 \\
Sling (36) & $49.1 \pm 27.0$ & $28.4 \pm 31.6$ & & $<0.001$ \\
No sling (20) & $36.7 \pm 33.6$ & $20.5 \pm 28.1$ & $25.7 \pm 29.0$ & 0.021 \\
Obstructive & & & $21.4 \pm 39.1$ & \\
Sling (35) & $44.0 \pm 33.6$ & $17.6 \pm 24.8$ & & \\
No sling (21) & $34.1 \pm 35.5$ & & & \\
\hline
\end{tabular}

Complications in the current trial may have been kept to a minimum secondary to this variable.

\section{Conclusion}

In conclusion, we have found the vaginal repair of anterior/ apical wall prolapse utilizing a lightweight soft type I anterior wall mesh placed via the Elevate system - a safe, minimally invasive, and effective procedure for the treatment of anterior wall prolapse in this subset of mostly postmenopausal patients. We feel that the role of mesh in vaginal repairs is in its infancy, and much study still needs to be done to determine the ideal material to be utilized and the optimal way to place and attach the graft vaginally and

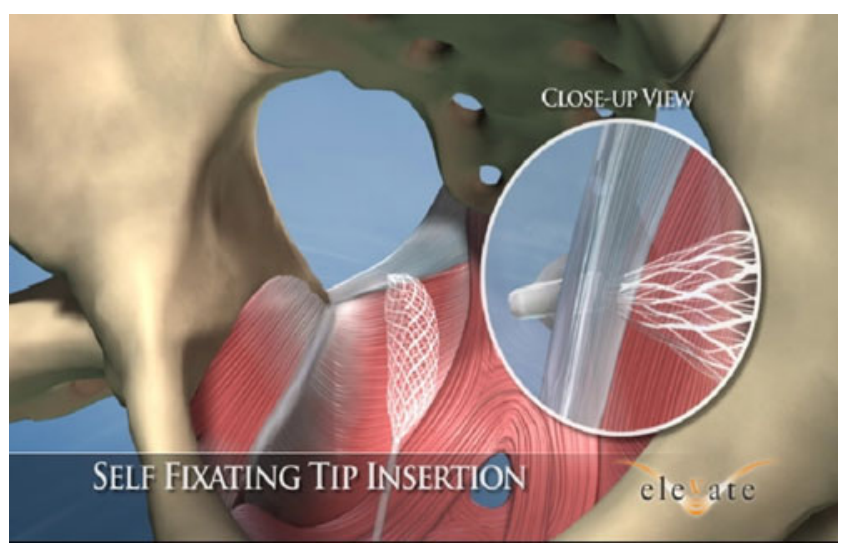

Fig. 2 Close-up view of the small self-fixating tip attaching into the ligament (Reproduced with permission from AMS) the proper patient to utilize it in; however, it can be expected that improvements in technology and techniques will continue to improve outcomes. The small self-fixating tips on the arms of the Elevate procedure that hold the mesh in place are one of the least invasive approaches to fixate a mesh graft in place vaginally to date. The elimination of trocar-based, blind needle passes through the groins also seems to decrease the risk of complications. We do recommend further prospective studies with longer-term follow-up to further help delineate its role in clinical practice.

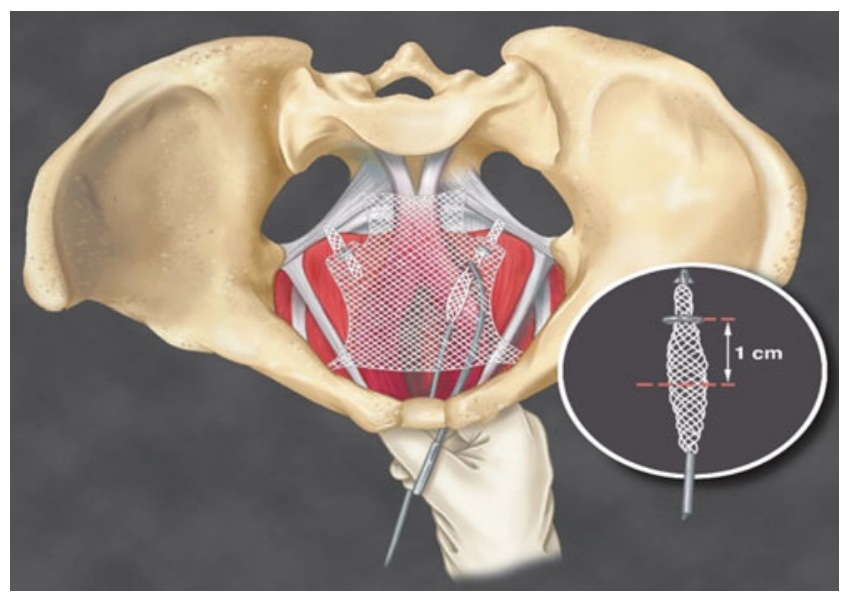

Fig. 3 Final adjustment of the graft into place. The bladder neck portion of the graft has been fixated to the levators and the apical portion of the graft is slid up the arms in a tension-free manner to elevate the anterior wall and vault. (Reproduced with permission from AMS) 
Conflicts of interest RD Moore is a consultant and speaker for the research grants of American Medical Systems (AMS). GK Mitchell has nothing to disclose. JR Miklos is a consultant of the research grants of AMS and a speaker for Coloplast. No funding, research grants, stipends, or any other outside assistance was received in support of the current paper.

Open Access This article is distributed under the terms of the Creative Commons Attribution Noncommercial License which permits any noncommercial use, distribution, and reproduction in any medium, provided the original author(s) and source are credited.

\section{References}

1. Beer M, Kuhn A (2005) Surgical techniques for vault prolapse: a review of the literature. Eur J Obstet Gynecol Reprod Biol 119:144-155

2. Olsen AL, Smith VJ, Bergstrom JO et al (1997) Epidemiology of surgically managed pelvic organ prolapse and urinary incontinence. Obstet Gynecol 8:501-506

3. Maher C, Baessler K (2006) Surgical management of anterior vaginal wall prolapse: an evidence based literature review. Int Urogynecol J Pelvic Floor Dysfunct 17:195-201

4. Nygaard IE, McReery R, Brubaker L (2004) Abdominal sacralcolpopexy: a comprehensive review. Obstet Gynecol 104(4):805-823

5. Maher C, Baessler K, Glazener CM et al (2008) Surgical management of pelvic organ prolapse:a short version Cochrane review. Neurourol Urodyn 27(1):3-12

6. Maher C, Feiner B, Baessler K, Glazener CMA. Surgical management of pelvic organ prolapse in women. Cochrane Database of Systematic Reviews 2010, Issue 4. Art. No.: CD004014. doi:10.1002/14651858.CD004014.pub4

7. Moore RD, Beyer RD, Jacoby K, Freedman SJ, McCammon KA, Gambla MT (2010) Prospective multicenter trial assessing type I, polypropylene mesh placed via transobturator route for the treatment of anterior vaginal prolapse with 2-year follow-up. Int Urogynecol J Pelvic Floor Dysfunct 21(5):545-552
8. Withagen MI, Milani AL, den Boon J, Vervest MD, Vierhout ME (2011) Trocar-guided mesh compared with conventional vaginal repair in recurrent prolapse:a randomized trial. Obstet Gynecol 117(2):242-250

9. Moore RD, Miklos JR (2009) Vaginal mesh kits for pelvic organ prolapse, friend or foe: a comprehensive review. Scientific World Journal 9:163-189

10. Sung VW, Rogers RG, Schaffer JI, Balk EM, Uhlig K, Lau J, Abed H, Wheeler TL 2nd, Morrill MY, Clemons JL, Rahn DD, Lukban JC, Lowenstein L, Kenton K, Young SB, Society of Gynecologic Surgeons Systematic Review Group (2008) Graft use in transvaginal pelvic organ prolapse repair: a systematic review. Obstet Gynecol 112(5):1131-1142

11. Altman D, Falconer C (2007) Perioperative morbidity using transvaginal mesh in pelvic organ prolapse repair. Obstet Gynecol 109(2 Pt 1):303-308

12. Margulies RU, Lewicky-Gaupp C, Fenner DE, McGuire EJ, Delancy JO (2008) Complications requiring reoperation following vaginal mesh kit procedures for prolapse. Am J Obstet Gynecol 199:678.e1-678.e4

13. Ridgeway B, Walters MD, Paraiso MF, Barber MD, McAchran SE, Goldman HB, Jelovsek (2008) Early experience with mesh excision for adverse outcomes after transvaginal mesh placement using prolapse kits. Am J Obstet Gynecol 199:703.e1-703.e7

14. Iglesia CB, Sokol AL, Sokol ER, Kudish BI, Gutman RE, Peterson JL, Shott S (2010) Vaginal mesh for prolapse: a randomized controlled trial. Obstet Gynecol 116(2 Pt 1):293-303

15. Goldberg RP, Tomezsko JE, Winkler HA, Koduri S, Culligan PJ, Sand PK (2001) Anterior or posterior sacrospinous vaginal vault suspension. Obstet Gynecol 98(2):199-204

16. Feiner B, Maher C (2010) Vaginal mesh contraction: definition, clinical presentation, and management. Obstet Gynecol 115(2 Pt 1):325-330

17. Moore RD, Mikos JR. Vaginal repair of cystocele with anterior wall mesh via transobturator route:efficacy and complications with up to 3-year follow-up. Adv Urol. 2009:743831. 2009 Aug 24 (in press)

18. Milani AL, Hinoul P, Gauld JM, Sikirica V, van Drie D, Cosson M, Prolift + M Investigators (2011) Trocar-guided mesh repair of vaginal prolapse using partially absorbable mesh: 1 year outcomes. Am J Obstet Gynecol 204(1):74.e 1-74.e8 